\title{
VITUS 3D Body Scanner
}

\author{
Markus MAURER* \\ Vitronic $\mathrm{GmbH}$, Wiesbaden, Germany
}

\begin{abstract}
For more than 15 years Vitronic has been a world leader in body scanning. Vitronic develops and manufactures body scanning systems for different areas of applications and to suit different international market.
\end{abstract}

Keywords: 3d body scanning, 3d body scanner

\section{Introduction}

Vitronic started development of this technology in 1994 when Vitronic agreed to create a prototype body scanner for a local artist. The idea was to take a 3-dimensional scan of a model and to have the rough version of this scan carved into wood. The design of this system was based on technology that Vitronic had been using in different industries in previous years, such as the 3 dimensional measurement of aluminium ingots, and other general machine vision applications which Vitronic has been selling since its inception in 1984. As the technology developed, Vitronic were able to improve on the initial design and started selling a finished product in 1997 called VITUS pro. Most customers of VITUS pro were (or still) using this scanner for research and anthropometrical measurements (for instance: BMW, DuPont, MiraLab, TNO).

In 1999 Vitronic finished the next body scanner development. This development was driven by the demands of the strong new markets for made-to-measure applications. This scanner was a smaller and cheaper body scanner called "VITUS smart". The VITUS smart scanner was designed for installation in a fitting room. This concept of single small scanning pillars still provides the basis for the latest body scanners. Today Vitronic offers two different versions of body scanners to best satisfy the differing requirements of various market segments.

VITUS smart LC is the entry-level model and the scanner is dedicated to applications were the requested floor space is crucial, for example, made to measure solutions in the retail environment.

VITUS smart XXL is the second body scanner model. The scanning volume of VITUS smart XXL complies with DIN EN ISO 20685 ("3-D scanning methodologies for internationally compatible anthropometric databases"). In addition, this scanner is designed to deliver high density data and minimize hidden surfaces during data acquisition.

Today hundreds of VITUS 3D body scanners are used for a large variety of applications.

\section{Method and Technical Key Factors}

The VITUS 3D Body Scanner utilizes integrated hardware and software components to perform the process of translating surfaces of physical objects into 3D dimensional data. The VITUS 3D Body Scanner uses the light stripe method as the basis for its measurements.

\subsection{Light stripe method}

The method used by VITUS is the light stripe method. The functional components of one scanning unit of the VITUS scanner are a diode laser with a cylindrical optical system and a matrix camera. The wavelength of the laser is inside the red band of the visible light spectrum. The laser is used to generate a structured illumination line and the camera is used as a sensor to capture an image of the laser in the target area.

\footnotetext{
*markus.maurer@vitronic.com; +49-611-7152-133; www.vitronic.com
} 


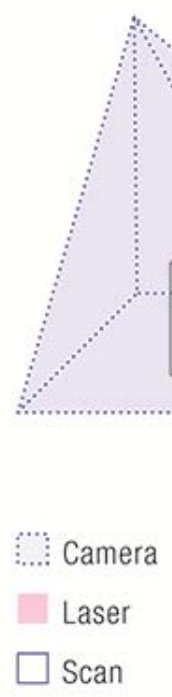

Fig. 1. Light Stripe Method.

The laser produces a line of light that illuminates the region of interest, mounted on a movable device while the object remains stationary. The camera is also mounted on the moveable device at a specific position with a defined angle relative to the laser axis. The camera, or sensor, detects the light reflected from the object. Changes in the shape of the object surface cause the distance from the light point to the sensor to fluctuate.

The sensor then maps this distance onto a plane using $\mathrm{x}$ and $\mathrm{y}$ components. By means of a calibration procedure the scanning unit is able to translate these distances in $\mathrm{mm}$ values. By combining several planes it becomes possible to create a 3-dimentional image of the object.

\subsection{Body Scanner Layout}

Multiple scanning units are required since it is impossible for one scanning unit to scan an entire body. Each scanning unit can "see" only those parts of the object that are directed towards the camera. Several scanning units therefore have to be combined to scan the object from different directions. Each scanning unit is integrated into a pillar which provides the necessary vertical movement.

A complete system includes in most cases 3 or 4 laser scanning pillars either permanently attached to a hard level floor, or bolted into an aluminium frame; a computer and monitor; an A/D signal converter box; and a calibration tube.

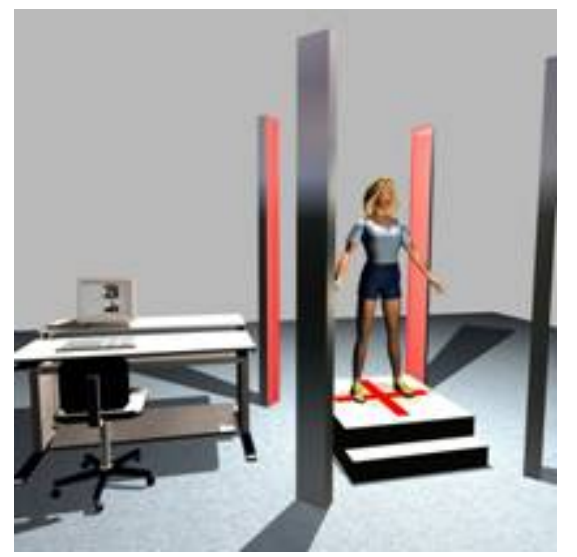

Fig. 2. Complete Body Scanning System [1].

The sensor units are initially arranged at the same horizontal level in each column and move from top to bottom in the columns to scan the entire body. Vertical movement is utilized to ensure that small movements of the body (i.e., postural sway) have a minimal effect on the measurement. 
A special calibration procedure ensures that the data delivered by from each of the sensors can be combined into a single coherent three-dimensional model of the scanned object.

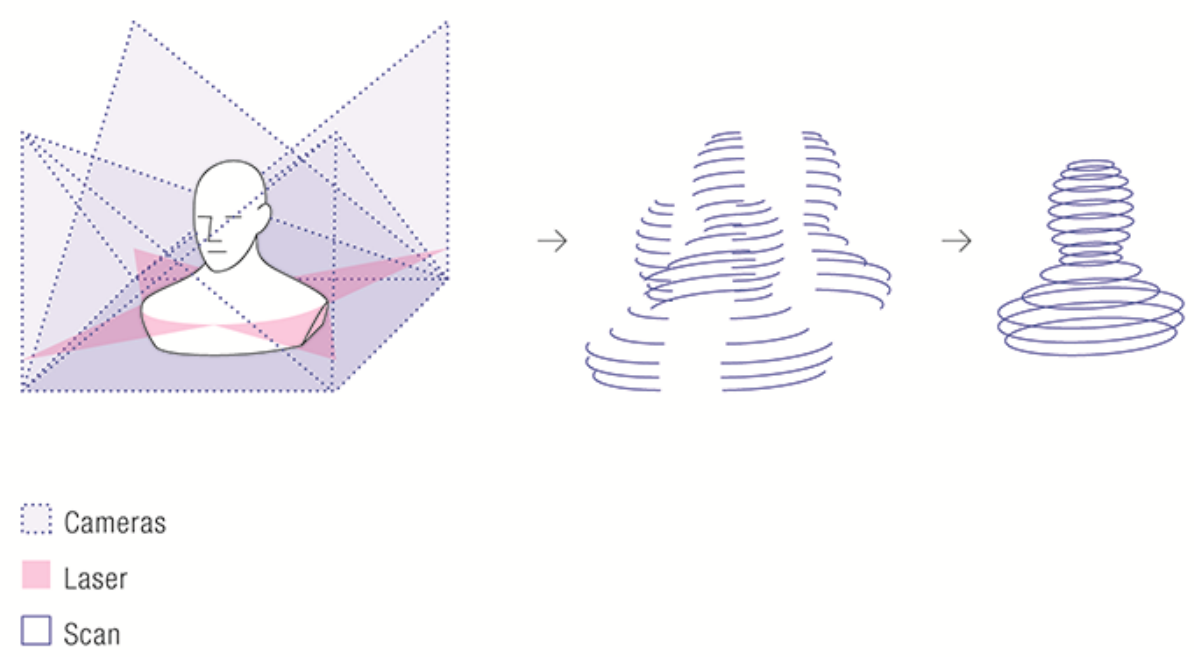

Fig. 3. Combination of different scans in a $3 d$ model by means of calibration.

\subsection{Occlusion}

After combining the output of several scanning units, there may be parts of the object that cannot be scanned due to occlusion, where the cameras do not "see" them. Assume, for example, that a set of scanning units is arranged around the object and moved linearly from top to bottom during the scanning process. If the light from the lasers forms a horizontal plane and the cameras are mounted above the lasers are directed slightly downward to record images of the laser line on the object, then it is impossible for the cameras to "see" the area below the horizontal laser line (e.g., under the chin of a standing person).

To avoid this situation, each sensor head can optionally be equipped with two cameras. Each sensor head then has one camera located above the laser plane, directed slightly downward and one camera located below the laser plane, directed slightly upwards. This duplication of the measurement area is called double triangulation and enables body areas such as the lower side of a chin to be scanned. Even when a body scanner employs double triangulation, there will still be some parts of an object where occlusion can occur and therefore may not be completely reconstructed.

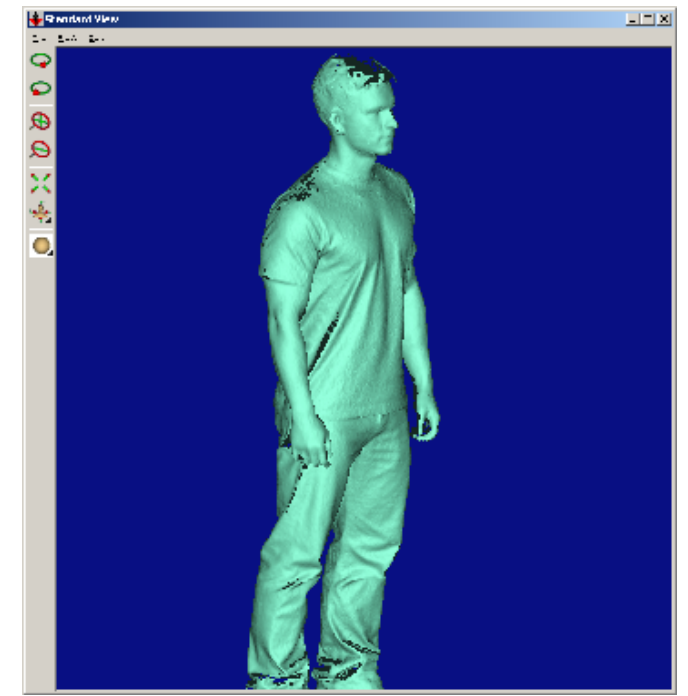

Fig.4. Occlusion of a body scan (e.g. wrinkles of the t-shirt). 
Although there is always the possibility to use software algorithms to try to reconstruct these gaps in the scanned data, one of the main goals for the layout of the VITUS body scanners was to find a set up were these occluded areas were minimized in the standard measurement positions.

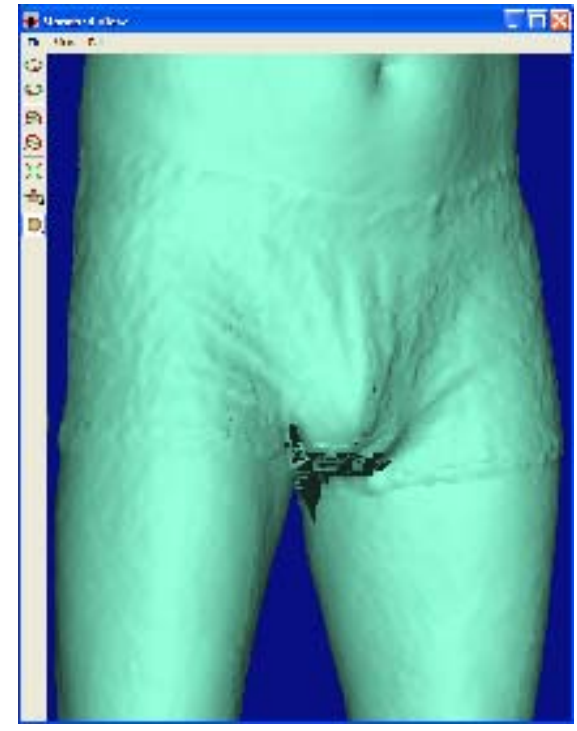

Fig. 5. Occluded areas in the row scan data.

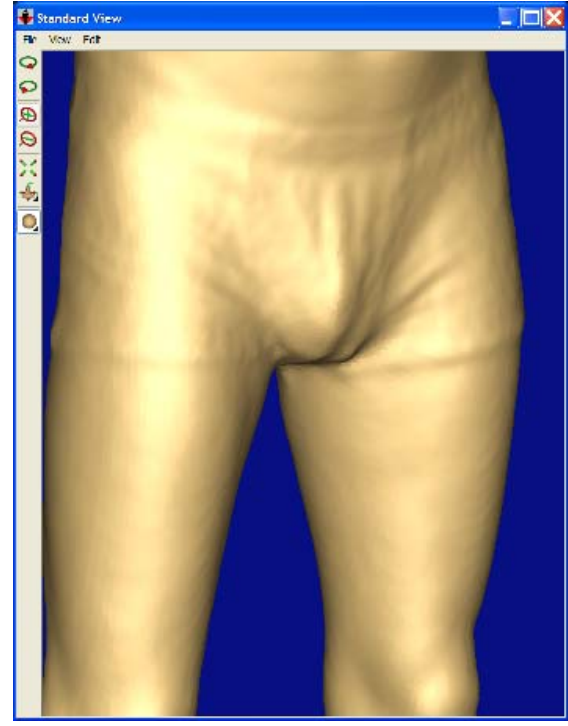

Fig. 6 Reconstructed $3 d$ model by software.

\subsection{Different Surface Colors}

As the measurement method is an optical one, all visible objects in the measurement area are included in the measurement result. However, if the surface is highly reflective or extremely dark, this could cause gaps in the signal and therefore degrade the result.

In general the light stripe method as deployed by the VITUS scanners is a very robust method and highly suitable for the task, because lasers of a specific wavelength are used to produce the light stripe. In addition the VITUS body scanners use a scanning unit with a very wide dynamic range so that different reflection intensities can be accurately detected.

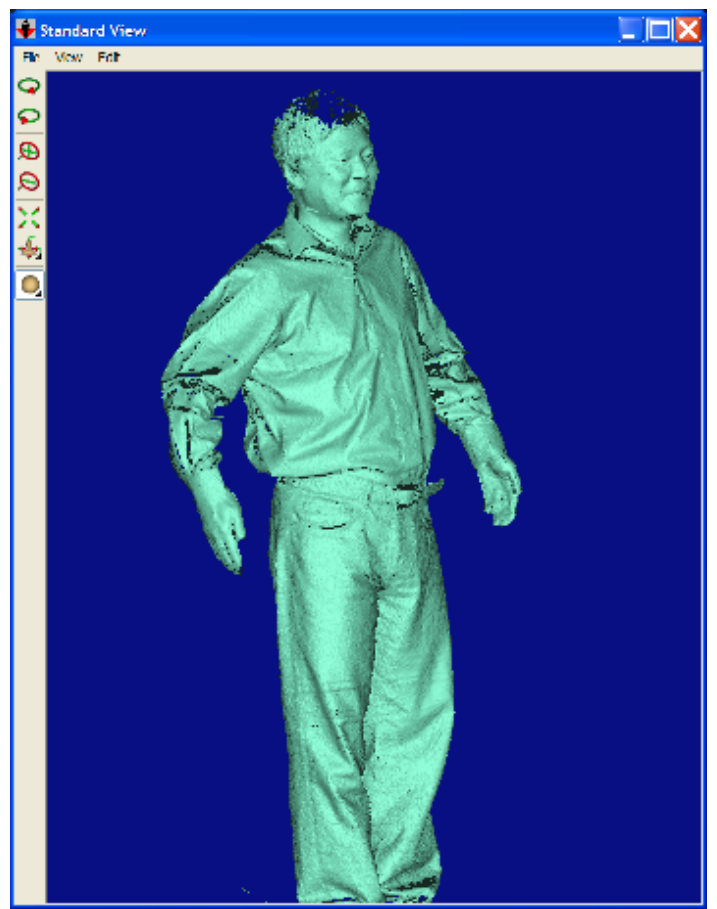

Fig.7. Scan of business man dressed in white shirt and black trousers. 


\subsection{Grey level Texture}

For some measurement applications it is beneficial to obtain the texture of the scanned point or surface area in addition to the $3 d$ surface point spatial information (i.e. $X, Y, Z$ value). This can be used for marker detection. The VITUS body scanners can therefore optionally provide grey level information of each scanned surface point.

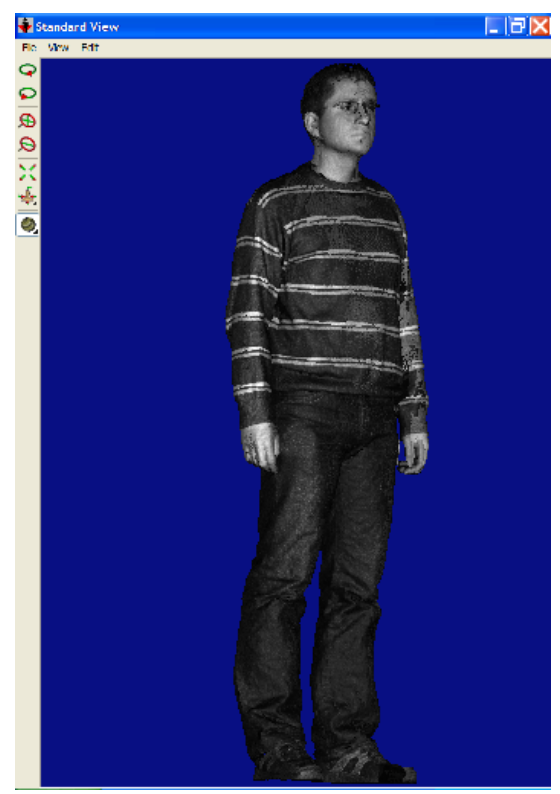

Fig.8. Grey level Scan.

\section{Cooperation with Human Solutions}

Vitronic entered into a partnership with "Human Solutions" in the early stages of the VITUS product family. Since 1999 "Human Solutions" and Vitronic have been cooperation partners. As an integrated solution partner, Human Solutions develops innovative solutions for customized clothing, size determination and anthropometrical measurements.

Today Human Solutions has extensive experience in this market. With more than 300 installations, Human Solutions is a worldwide leader in this market. Their solutions are based on VITUS body scanners (VITUS smart LC and VITUS smart XXL) for measurement of the customers.

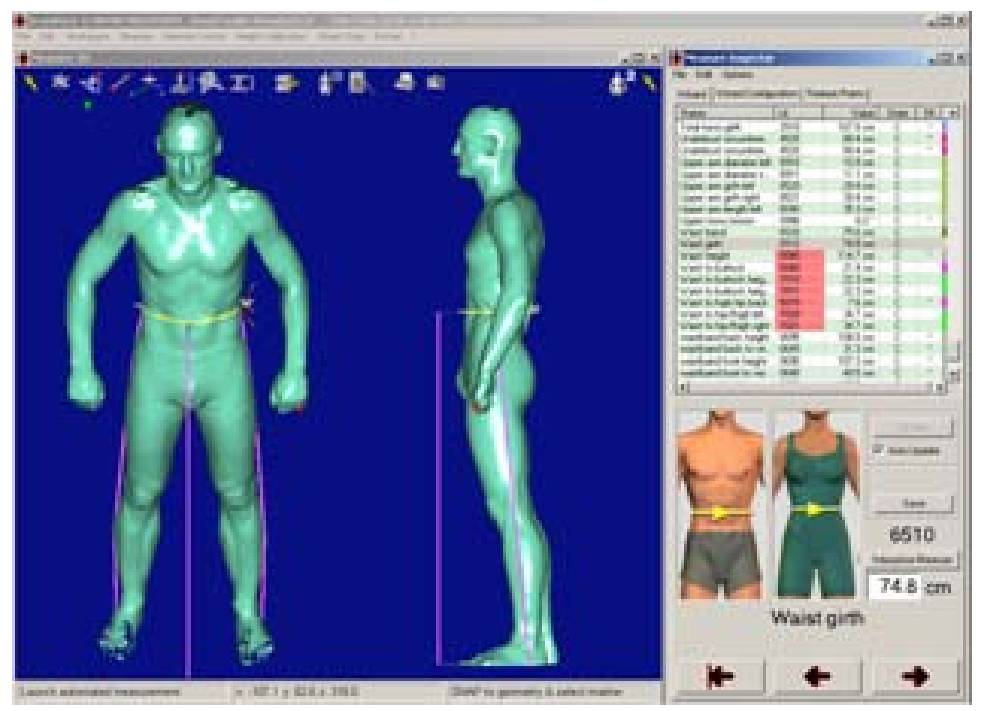

Fig.9. Typical application for measurement of the human body [1]. 


\section{VITUS smart body scanners}

\subsection{VITUS smart XXL}

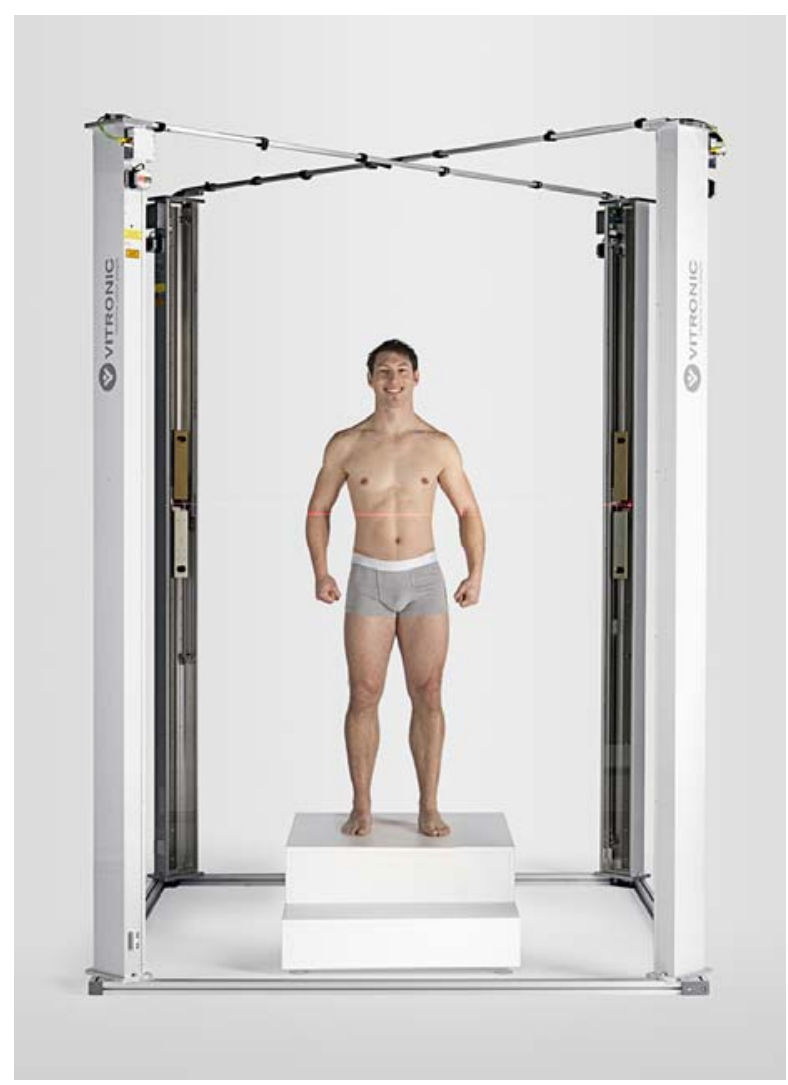

Fig.10.VITUS smart XXL.

Table 1. Specification VITUS smart XXL.

\begin{tabular}{|c|c|}
\hline Measurement principle & $\begin{array}{l}\text { optical triangulation } \\
\text { with laser light }\end{array}$ \\
\hline Safety & Eye-safe Laser Class 1 \\
\hline Sensor heads & 4 dual-camera heads \\
\hline Measurement range Height $\mathbf{Z}$ & $2100 \mathrm{~mm}$ \\
\hline Measurement range Depth X & $1000 \mathrm{~mm}$ \\
\hline Measurement range Width $\mathrm{Y}$ & $1200 \mathrm{~mm}$ \\
\hline $\begin{array}{l}\text { Accuracy } \\
\text { cylindrical tube } 110 \mathrm{~mm} \text { diameter, } \\
2100 \mathrm{~mm} \text { height constant } \\
\text { temperature within the range of } 15^{\circ}- \\
30^{\circ} \mathrm{C}\end{array}$ & Average girth error $<1 \mathrm{~mm}$ \\
\hline Measurement time approx. & $12 \mathrm{~s}$ \\
\hline Point density & $27 \mathrm{pts} / \mathrm{cm}^{2}$ \\
\hline Scanner Height Z & $2850 \mathrm{~mm}+$ Frame $100 \mathrm{~mm}$ \\
\hline Scanner Depth X & $2200 \mathrm{~mm}$ \\
\hline Scanner Width $\mathrm{Y}$ & $2200 \mathrm{~mm}$ \\
\hline Scanner Area & $4.84 \mathrm{~m}^{2}$ \\
\hline Input Voltage & $\begin{array}{l}230 \mathrm{~V} / 50 \mathrm{~Hz} \\
115 \mathrm{~V} / 60 \mathrm{~Hz} \\
420 \mathrm{VA}\end{array}$ \\
\hline
\end{tabular}




\subsection{VITUS smart LC}

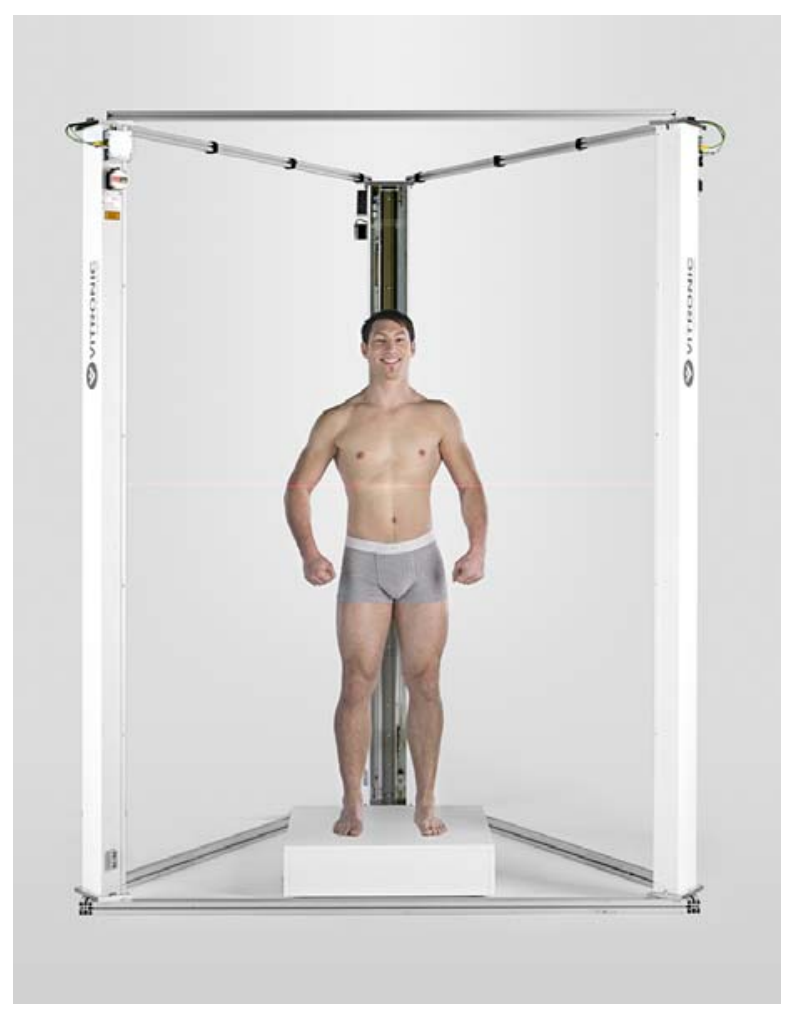

Fig.11 VITUS smart LC.

Table 2. Specification VITUS smart LC.

\begin{tabular}{|c|c|}
\hline Measurement principle & $\begin{array}{l}\text { optical triangulation } \\
\text { with laser light }\end{array}$ \\
\hline Safety & Eye-safe Laser Class 1 \\
\hline Sensor heads & 3 single-camera heads \\
\hline Measurement range Height Z & $2100 \mathrm{~mm}$ \\
\hline $\begin{array}{l}\text { Measurement range } \\
(\text { Triangle Base } X)\end{array}$ & $900 \mathrm{~mm}$ \\
\hline $\begin{array}{l}\text { Measurement range } \\
\text { (Triangle Width Y) }\end{array}$ & $900 \mathrm{~mm}$ \\
\hline $\begin{array}{l}\text { Accuracy } \\
\text { cylindrical tube } 110 \mathrm{~mm} \text { diameter, } \\
2100 \mathrm{~mm} \text { height constant } \\
\text { temperature } \\
\text { within the range of } 15^{\circ}-30^{\circ} \mathrm{C}\end{array}$ & average girth error $<3 \mathrm{~mm}$ \\
\hline Measurement time approx. & $12 \mathrm{~s}$ \\
\hline Point density & $7 \mathrm{pts} / \mathrm{cm} 2$ \\
\hline Scanner Height & $2500 \mathrm{~mm}+$ Frame $100 \mathrm{~mm}$ \\
\hline Scanner Base, Side length Triangle & $2200 \mathrm{~mm}$ \\
\hline Scanner Area (Triangle) & $2.98 \mathrm{~m} 2$ \\
\hline Input Voltage & $\begin{array}{l}230 \mathrm{~V} / 50 \mathrm{~Hz} \\
115 \mathrm{~V} / 60 \mathrm{~Hz} \\
420 \mathrm{VA}\end{array}$ \\
\hline
\end{tabular}

\section{References}

1. Pictures: Copyright by Human Solutions www.human-solutions.de. 